\title{
Perfil sensorial de bolos de chocolate formulados com farinha de yacon (Smallanthus sonchifolius)
}

\author{
Sensorial profile of chocolate cakes formulated with yacon flour (Smallanthus sonchifolius) \\ Vivianne Montarroyos PADILHA ${ }^{1 *}$, Priscilla Moura ROLIM ${ }^{1}$, Silvana Magalhães SALGADO로 \\ Alda Souza LIVERA ${ }^{1}$, Samara Alvachian Cardoso ANDRADE ${ }^{1}$, Nonete Barbosa GUERRA ${ }^{1}$
}

\begin{abstract}
Resumo
Neste trabalho foi avaliado o uso de farinha de yacon na formulação de bolos de chocolate para se verificar a influência deste constituinte sobre a qualidade sensorial e cromaticidade do produto. Foram analisadas amostras de bolos, formulados com farinha de yacon, denominadas: amostra P (bolo padrão, sem farinha de yacon), amostra A (bolo com $20 \%$ de farinha de yacon) e amostra B (bolo com $40 \%$ de farinha de yacon). Para a avaliação sensorial, foi realizada a Análise Descritiva Quantitativa (ADQ), utilizando 15 provadores treinados, os quais definiram 9 atributos sensoriais (cor, estrutura do miolo, aroma, gosto doce, sabor de chocolate, sabor residual, umectância, maciez e qualidade global). A análise física dos bolos foi realizada através da caracterização cromática. Os dados sensoriais foram submetidos à análise de variância ANOVA, sendo realizado o teste de Duncan para comparação entre as médias de Duncan, ao nível de 5\% de significância. Para verificar as correlações entre as amostras e os atributos, foi realizada a Análise de Componentes Principais (ACP). Na ACP, a soma de componentes principais 1 e 2 foi de $83,3 \%$, ou seja, a variabilidade entre as amostras foi explicada por estes dois componentes. Os resultados obtidos demonstraram que a adição de $40 \%$ de farinha de yacon resultou em maiores notas para o aroma $(10,16)$, gosto doce $(7,60)$, sabor de chocolate $(10,52)$, maciez $(11,44)$ e qualidade global $(11,64)$. Com relação ao atributo estrutura não houve diferença significativa entre as notas dos bolos $\mathrm{P}$, A e B. Independente da quantidade de farinha de yacon adicionada, o perfil sensorial dos bolos de chocolate foi influenciado e, em particular, o bolo com maior teor de farinha de yacon apresentou as melhores notas.

Palavras-chave: análise sensorial; prebióticos; análise de componente principal.
\end{abstract}

\begin{abstract}
This study evaluated the use of yacon flour in the formulation of chocolate cakes by investigating its influence on the sensory quality and chromaticity of the product. The following samples were prepared and analyzed: sample P (standard cake without yacon flour), sample A ( $20 \%$ of yacon flour), and sample B ( $40 \%$ of yacon flour). The Quantitative Descriptive Analysis (QDA) was used for the sensory analysis with 15 trained panelists who evaluated 9 sensory attributes (color, crumb structure, aroma, sweet taste, chocolate flavor, residual taste, humectancy, tenderness, and overall quality). The physical analysis of the cake was made by color characterization. The data were subjected to sensory analysis of variance ANOVA and the Duncan's test for comparison between the averages, at $5 \%$ level of significance. To verify the correlations between the samples and attributes, the Principal Components Analysis (PCA) was performed. In the PCA, the sum of the principal components 1 and 2 was $83.3 \%$, i.e., the variability between the samples was explained by these two components. The results showed that the addition of $40 \%$ of yacon flour resulted in higher scores for aroma (10.16), sweet taste (7.60), chocolate flavor (10.52), tenderness (11.44), and overall quality (11.64). With regard to the attribute crumb structure, there was no significant difference between the scores of cakes P, A, and B. Regardless of the amount of yacon flour added, the sensory profile of the chocolate cakes was influenced. Particularly, the cake with the highest content of yacon flour was attributed the highest scores.

Keywords: sensory análise; prebiotics; principal component analysis.
\end{abstract}

\section{Introdução}

Os frutanos (inulina e fruto-oligossacarídeos) caracterizamse pelos enlaces $\beta(2 \rightarrow 1)$ entre as unidades de frutose com molécula terminal de frutose, glicose ou sacarose. Apresentam a estrutura linear com diferente grau de polimerização (Gp). A inulina é um composto de maior $\mathrm{Gp}$, enquanto o oligofrutose e o fruto-oligossacarídeo, ambos de menor Gp, originam-se a partir da hidrólise da inulina e da transfructosilação da sacarose, respectivamente (BIEDRZYCKA; BIELECKA, 2004).

O notável interesse pelos frutanos advém do fato desses compostos serem resistentes às enzimas digestivas e, portanto, não digeridos pelo organismo humano. Consequentemente, chegam ao intestino grosso intactos e são hidrolisados e fermentados pelas bactérias anaeróbicas presentes no cólon (ROBERFROID, 2007).

Desta maneira, estes compostos são considerados fibras alimentares e têm impacto na indústria de alimentos devido às suas propriedades prebióticas e características tecnológicas como substituto do açúcar e da gordura, originando produtos de baixo valor calórico com características sensoriais satisfatórias (FRANCK, 2002).

${ }^{1}$ Nutrição, Universidade Federal de Pernambuco - UFPE, CEP 50670-901, Recife - PE, Brasil, E-mail: vivianne_padilha@yahoo.com.br

${ }^{*}$ A quem a correspondência deve ser enviada 
Os frutanos vêm sendo utilizados como ingredientes na formulação de produtos lácteos, congelados, forneados e cárnicos: a inulina por suas propriedades similares ao amido, ou seja, a formação de géis estáveis, e os FOS pela sua função umectante e edulcorante semelhante à sacarose, porém com aporte calórico reduzido (1,5 kcal.g ${ }^{-1}$ ) (ROBERFROID, 2002; RODRÍGUEZ et al., 2006).

Os frutanos são encontrados em quantidades expressivas em alimentos como, cebola, banana, alcachofra, alho, raízes de almeirão, chicória e raízes de yacon (HARTEMINK; VANLAERE; ROMBOUTS, 1997; GOTO et al., 1995; SILVA et al., 2007).

Atualmente se produzem em escala comercial misturas de frutanos principalmente a partir da raiz da chicória. Além desta, o yacon, está sendo considerado uma cultura com um potencial promissor como fonte de frutanos. O yacon (Smallanthus sonchifolius), planta herbácea perene originalmente cultivada na região dos Andes na América do Sul, foi introduzido no Brasil no início dos anos 90 (HONG et al., 2008). Possui estocado em suas raízes tuberosas cerca de $90 \%$ de carboidratos (base seca), sendo 40 a $70 \%$ correspondente aos frutanos, principalmente fruto-oligossacarídeos (MANRIQUE; PÁRRAGA, 2005; MADRIGAL; SANGRONIS, 2007).

Os frutanos vêm sendo incorporados em diversos produtos alimentares, principalmente em produtos de padaria e confeitaria, como bolo, que têm grande aceitação pelo mercado consumidor devido às suas características reológicas: produtos leves e facilmente mastigáveis.

Sabendo dos efeitos fisiológicos dos frutanos e a crescente demanda por alimentos que apresentem, além da alta qualidade sensorial e nutricional, benefícios associados à saúde, surge à necessidade de formular alimentos adicionados de ingredientes com alegações funcionais.

Diante destas constatações, o presente trabalho objetivou utilizar a farinha de yacon na formulação de bolo de chocolate, avaliando a influência deste constituinte sobre a qualidade sensorial e caracterização cromática do produto.

\section{Material e métodos}

\subsection{Material}

Processo de obtenção da farinha de yacon

As raízes de yacon (Smallanthus sonchifolius) in natura foram obtidas no CEASA (Centro de Abastecimento da Região Metropolitana do Recife - PE) na Região Metropolitana do Recife. Inicialmente as raízes foram lavadas em água corrente e sanitizadas em solução clorada 200 ppm por 10 minutos. Posteriormente foram descascadas e cortadas em lâminas finas em multiprocessador. As lâminas de yacon foram imersas em uma solução de cloreto de cálcio a $1 \%$ por 30 minutos. Por fim, foram submetidas à secagem em estufa ventilada a $55^{\circ} \mathrm{C}$ por 24 horas e trituradas em microprocessador para a obtenção da farinha com granulometria de 20 mesh (Figura 1).

\section{Preparação dos bolos}

Os bolos foram formulados conforme a Tabela 1 , seguindo a metodologia citada por Moscatto et al. (2006), salvo algumas modificações.

\subsection{Métodos}

\section{Determinação do perfil sensorial}

Pré-seleção dos provadores

Os candidatos foram submetidos ao teste de sensibilidade aos sabores básicos (Teste Threshold de Reconhecimento). De vinte provadores recrutados voluntariamente, foram préselecionados quinze provadores, pela habilidade em discriminar a intensidade dos quatro sabores (amargo, doce, salgado e ácido) com o mínimo de 70\% de respostas certas das amostras oferecidas (ANZALDUA-MORALES, 1994).

\subsection{Análise Descritiva Quantitativa (ADQ)}

Uma ampla lista de termos descritores foi levantada na literatura pelos pesquisadores (DUTCOSKY, 2007). Sob a supervisão do líder da equipe, os provadores discutiram o significado de cada termo e definiram de forma consensual nove termos que melhor caracterizavam bolo de chocolate. As definições dos atributos descritivos para as amostras de bolo estão apresentadas na Tabela 2. A análise sensorial foi realizada por meio do Teste ADQ (BRASIL, 2005).

\subsection{Avaliação das amostras}

Os provadores, mediante termo de consentimento livre e esclarecido, foram convocados a participar da avaliação das amostras. Foi elaborada a Ficha de Análise Sensorial dos bolos (Figura 2) com escalas não estruturadas de $15 \mathrm{~cm}$, ancoradas

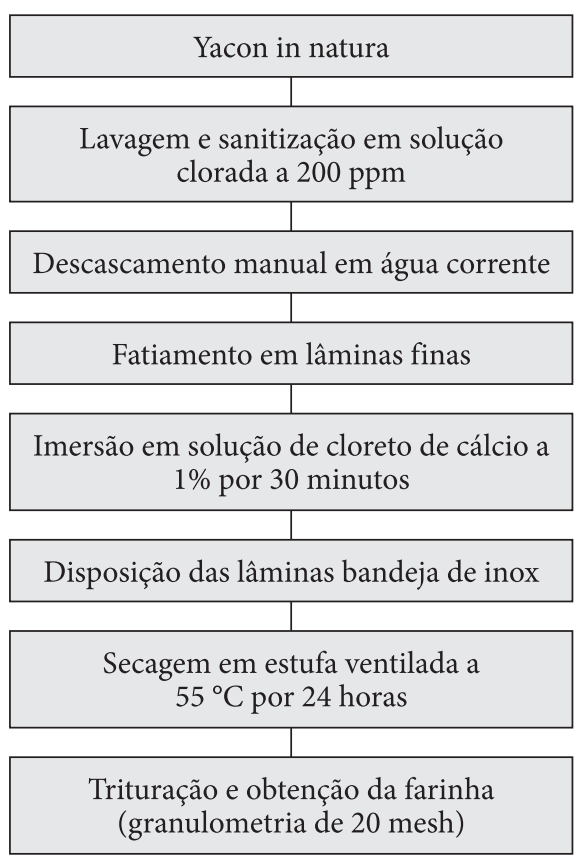

Figura 1. Processamento do yacon para obtenção da farinha. 
nos pontos extremos com palavras que indicam intensidade para avaliar a intensidade dos atributos sensoriais presentes nas amostras.

$\mathrm{Na}$ aplicação dos testes sensoriais, as fatias de bolo foram servidas em pratos brancos codificados com números de três dígitos, em blocos completos balanceados, com três repetições, acompanhados de copos de água em temperatura ambiente. Para avaliação do aroma, foram oferecidos aos provadores recipientes fechados, contendo pedaços dos bolos em estudo. Os testes foram realizados em cabines individuais no Laboratório de Análise Sensorial do Departamento de Nutrição, da Universidade Federal de Pernambuco - UFPE. Os julgadores foram orientados a provar as amostras da esquerda para a direita.

\subsection{Análise estatística}

Os dados sensoriais foram submetidos à análise de variância (ANOVA), sendo realizado o teste de Duncan para comparação entre as médias obtidas, ao nível de $5 \%$ de significância. Para verificar as correlações entre as amostras e os atributos sensoriais, foi realizada a Análise de Componente Principal (ACP) (MINIM, 2006). Utilizou-se o software statistic for Windows (STATSOFT, 2002).

\section{Análise instrumental de cor}

A determinação dos bolos foi efetuada no Laboratório de Experimentação e Análise de Alimentos Nonete Barbosa Guerra (LEAAL), utilizando colorímetro marca Minolta CR-400 (Konica Minolta Sensing, Inc.). As cores das amostras dos bolos foram avaliadas através de três parâmetros, o CIE L ${ }^{*}$ $\mathrm{a}^{*} \mathrm{~b}^{*}$ (Comissão Internacional de Iluminantes). O parâmetro $\mathrm{L}^{*}$ define a luminosidade $\left(\mathrm{L}^{*}=0\right.$ preto $\mathrm{e}^{*}=100$ branco) e $\mathrm{a}^{*} \mathrm{e} \mathrm{b}^{\star}$ são responsáveis pela cromaticidade $\left(+\mathrm{a}^{*}\right.$ vermelho $\mathrm{e}-\mathrm{a}^{\star}$ verde, $+\mathrm{b}^{\star}$ amarelo e $-\mathrm{b}^{\star}$ azul). Para cada tratamento, as determinações foram efetuadas em triplicata e os resultados expressos como média (MCGUIRE, 1992).

\section{Resultados e discussão}

\subsection{Análise Descritiva Quantitativa (ADQ)}

A partir dos atributos escolhidos em consenso pelos membros da equipe sensorial foi efetuada a caracterização do perfil sensorial das amostras, cujas médias de cada atributo avaliado encontram-se na Tabela 3.
Com relação ao bolo com $40 \%$ de farinha de yacon, foram detectadas as maiores médias para os atributos aroma, gosto doce, maciez, sabor de chocolate e qualidade global, sendo apenas os três últimos atributos significativamente diferentes das demais amostras. Ainda com relação à amostra $\mathrm{B}, \mathrm{o}$ atributo maciez alcançou a maior média, possivelmente pela presença de

\begin{tabular}{|c|c|}
\hline \multicolumn{2}{|c|}{ Ficha ADQ } \\
\hline Nome: & Data: \\
\hline Código da & \\
\hline \multicolumn{2}{|c|}{$\begin{array}{l}\text { Por favor, escreva seu nome, data e código da amostra na folha } \\
\text { de avaliação. Analise cada amostra e preencha as respostas na } \\
\text { sequência em que aparecem em sua ficha de avaliação, fazendo } \\
\text { um traço vertical na linha, na posição (ponto) que melhor reflita } \\
\text { seu julgamento. Prove quantidade suficiente de amostra e dispo- } \\
\text { nha do tempo necessário para avaliar cada característica. Por } \\
\text { favor, enxágue a boca após a avaliação de cada amostra. }\end{array}$} \\
\hline \multicolumn{2}{|c|}{ Característica: } \\
\hline \multicolumn{2}{|l|}{ Aroma } \\
\hline Fraco & Forte \\
\hline \multicolumn{2}{|c|}{ Cor marron do miolo } \\
\hline Claro & $\overline{\text { Escuro }}$ \\
\hline \multicolumn{2}{|c|}{ Estrutura do miolo } \\
\hline Leve & $\overline{\text { Pesada }}$ \\
\hline \multicolumn{2}{|l|}{ Gosto doce } \\
\hline Fraco & Forte \\
\hline \multicolumn{2}{|c|}{ Sabor característico de chocolate } \\
\hline Fraco & Forte \\
\hline \multicolumn{2}{|c|}{ Sabor residual } \\
\hline Fraco & Forte \\
\hline \multicolumn{2}{|l|}{ Maciez } \\
\hline Pouco & Muito \\
\hline \multicolumn{2}{|c|}{ Umectância } \\
\hline Pouco & Muito \\
\hline \multicolumn{2}{|c|}{ Qualidade global } \\
\hline Baixa & Alta \\
\hline
\end{tabular}

Figura 2. Ficha de Análise Sensorial dos bolos.

Tabela 1. Proporção dos ingredientes nas formulações dos bolos.

\begin{tabular}{|c|c|c|c|}
\hline \multirow[t]{2}{*}{ Ingredientes } & \multicolumn{3}{|c|}{ Formulações } \\
\hline & Amostra padrão $(\mathrm{P}) \%$ & Amostra experimental A(\%) & Amostra experimental B (\%) \\
\hline Ovos médios & 2 unidades & 2 unidades & 2 unidades \\
\hline Margarina (80\% de lipídeos) & $100 \mathrm{~g}$ & $100 \mathrm{~g}$ & $100 \mathrm{~g}$ \\
\hline Leite em pó reconstituído & $100 \mathrm{~mL}$ & $90 \mathrm{~mL}$ & $80 \mathrm{~mL}$ \\
\hline Açúcar cristal & $100 \mathrm{~g}$ & $70 \mathrm{~g}$ & $40 \mathrm{~g}$ \\
\hline Cacau em pó & $6 \mathrm{~g}$ & $6 \mathrm{~g}$ & $6 \mathrm{~g}$ \\
\hline Achocolatado & $36 \mathrm{~g}$ & $36 \mathrm{~g}$ & $36 \mathrm{~g}$ \\
\hline Farinha de trigo & $100 \mathrm{~g}$ & $80 \mathrm{~g}$ & $60 \mathrm{~g}$ \\
\hline Farinha de yacon & - & $20 \mathrm{~g}$ & $40 \mathrm{~g}$ \\
\hline Fermento em pó & $16 \mathrm{~g}$ & $16 \mathrm{~g}$ & $16 \mathrm{~g}$ \\
\hline Sal & $3 \mathrm{~g}$ & $3 \mathrm{~g}$ & $3 \mathrm{~g}$ \\
\hline
\end{tabular}


frutanos (inulina) que, ao entrar em contato com o leite, forma uma mistura cremosa conferindo uma sensação tátil bucal semelhante à gordura (ZULETA; SAMBUCETTI, 2006).

Quanto ao atributo gosto doce, o bolo B, formulado com menor teor de açúcar refinado (40 g) e maior teor de farinha de yacon (40\%), apresentou a maior nota, salientando-se que não diferiu significativamente $(\mathrm{p}<0,05)$ da amostra padrão e comprovando a atuação dos FOS como substituto de açúcar (SILVA et al., 2007 ).

A Figura 3 demonstra o perfil sensorial dos produtos analisados, salientando suas similaridades e diferenças. $\mathrm{O}$ centro da figura representa o ponto zero da escala e a intensidade aumenta do centro para a periferia. A média de cada atributo por amostra é marcada no eixo correspondente, cujo perfil sensorial é traçado pela conexão dos pontos. De acordo com a Tabela 3, pode-se verificar que apenas para o atributo estrutura não houve diferença significativa entre as amostras $(\mathrm{p}<0,05)$; para este último atributo, a amostra $\mathrm{B}$ obteve a menor nota.

Tabela 2. Definições dos atributos descritivos para as amostras de bolo.

\begin{tabular}{|c|c|}
\hline Descritores & Definições \\
\hline Aroma & $\begin{array}{l}\text { Aroma de bolo preparado com achocolatado e } \\
\text { cacau em pó }\end{array}$ \\
\hline Cor do miolo & Cor característica do miolo do bolo \\
\hline Estrutura do miolo & $\begin{array}{l}\text { Atributo de aparência em relação à estrutura do } \\
\text { centro do bolo }\end{array}$ \\
\hline Gosto doce & Sabor característico de sacarose \\
\hline Sabor de chocolate & $\begin{array}{l}\text { Sabor de bolo preparado com achocolatado e } \\
\text { cacau em pó }\end{array}$ \\
\hline Sabor residual & $\begin{array}{l}\text { Sensação olfatogustativa que ocorre após } \\
\text { degustação do bolo devido a presença de algum } \\
\text { ingrediente que esteja em maior evidência na } \\
\text { formulação }\end{array}$ \\
\hline Maciez & $\begin{array}{l}\text { Força necessária para provocar uma determina- } \\
\text { da deformação }\end{array}$ \\
\hline Umectância & $\begin{array}{l}\text { Sensação provocada pela quantidade de água } \\
\text { no alimento }\end{array}$ \\
\hline Qualidade global & $\begin{array}{l}\text { Características gerais que determinam o perfil } \\
\text { sensorial do alimento }\end{array}$ \\
\hline
\end{tabular}

Tabela 3. Médias da equipe para os termos descritores de bolo de chocolate.

\begin{tabular}{lccc}
\hline Atributos sensoriais & Amostra P & Amostra A & Amostra B \\
\hline Aroma & $9,81^{\mathrm{b}}$ & $10,00^{\mathrm{ab}}$ & $10,16^{\mathrm{a}}$ \\
Cor do miolo & $11,52^{\mathrm{a}}$ & $10,58^{\mathrm{b}}$ & $10,65^{\mathrm{b}}$ \\
Estrutura do miolo & $7,59^{\mathrm{a}}$ & $7,87^{\mathrm{a}}$ & $7,23^{\mathrm{a}}$ \\
Gosto doce & $7,27^{\mathrm{a}}$ & $6,47^{\mathrm{b}}$ & $7,60^{\mathrm{a}}$ \\
Sabor de chocolate & $9,69^{\mathrm{c}}$ & $10,13^{\mathrm{b}}$ & $10,52^{\mathrm{a}}$ \\
Sabor residual & $4,83^{\mathrm{a}}$ & $3,57^{\mathrm{b}}$ & $3,58^{\mathrm{b}}$ \\
Maciez & $10,82^{\mathrm{b}}$ & $10,52^{\mathrm{b}}$ & $11,44^{\mathrm{a}}$ \\
Umectância & $9,85^{\mathrm{a}}$ & $9,94^{\mathrm{a}}$ & $8,56^{\mathrm{b}}$ \\
Qualidade global & $11,07^{\mathrm{b}}$ & $10,62^{\mathrm{c}}$ & $11,64^{\mathrm{a}}$ \\
\hline
\end{tabular}

Médias seguidas de letras iguais na horizontal não diferem significativamente ao nível de 5\% de significância pelo teste de Duncan; Amostra P = Bolo Padrão; Amostra A = Bolo Experimental A ( $20 \%$ de farinha de yacon); Amostra B = Bolo Experimental B ( $40 \%$ de farinha de yacon)
O gráfico dos Componentes Principais (ACP) (Figura 4) ilustra as correlações entre as amostras e os atributos sensoriais. Cada amostra é representada por um triângulo, sendo que cada vértice é definido pelas repetições atribuídas pela equipe sensorial.

As amostras foram caracterizadas pelos vetores que se apresentam mais próximos a elas. Neste estudo, os dois componentes principais explicaram $83,3 \%$ da variabilidade total obtida entre os bolos, ou seja, a variabilidade entre as amostras pôde ser bem explicada utilizando apenas esses dois eixos.

Ao analisar o primeiro componente principal (Figura 4) que reproduz 54,29\%, constatou-se que a amostra B possuiu escores mais positivos, sendo, portanto, melhor representada pelos atributos sensoriais, qualidade global, maciez e sabor de chocolate, fato esse confirmado pelas maiores e significativas notas $(\mathrm{p}<0,05)$ obtidas por estes atributos (Tabela 3 ). Diferentemente, das amostras A e P apresentaram escores negativos, sendo caracterizadas pelo atributo umectância, ou seja, apresentaram notas significativamente maiores que a amostra B.
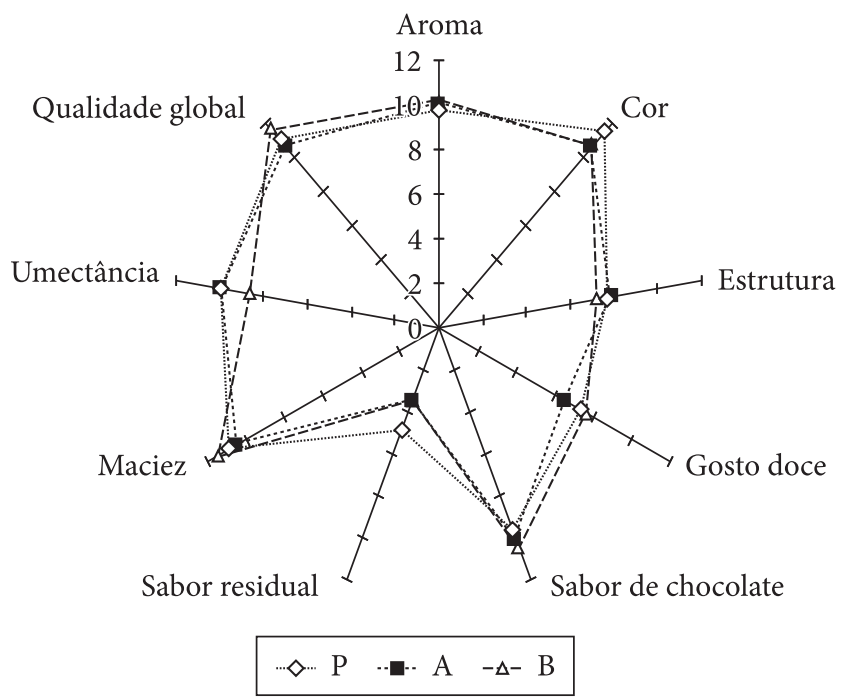

Figura 3. Perfil sensorial dos bolos de chocolate.

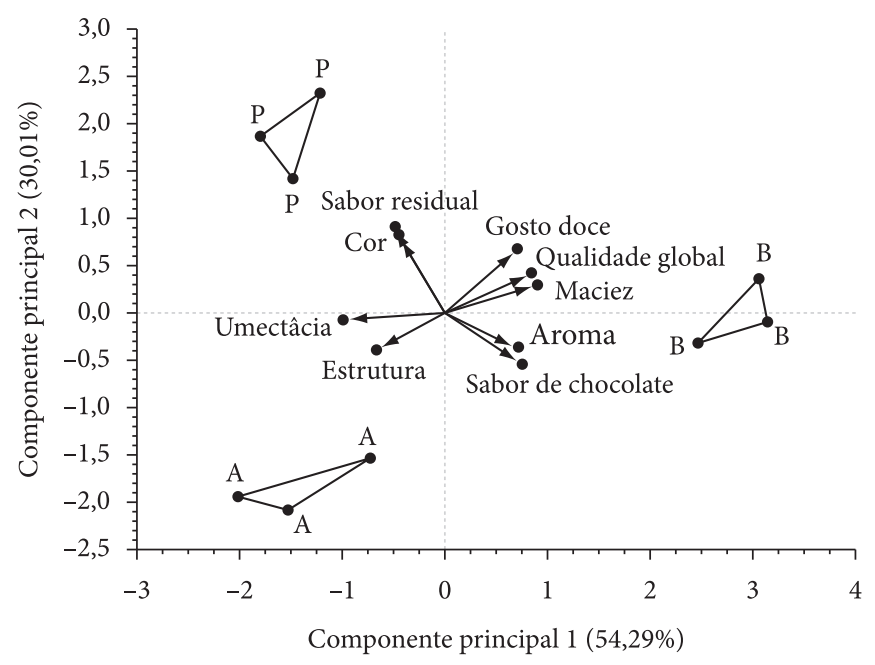

Figura 4. Análise dos Componentes Principais (ACP). 
O segundo componente principal (Figura 4) reproduz $30,01 \%$, constatando-se que a amostra $\mathrm{P}$ possuiu escores mais positivos, sendo caracterizada pelos atributos sabor residual e cor do miolo. Esse resultado pode ser confirmado pela Tabela 3, onde as médias da amostra $\mathrm{P}$ para os dois atributos foram significativamente $(\mathrm{p}<0,05)$ maiores que as encontradas para as amostras A e B.

\subsection{Caracterização cromática das amostras}

$\mathrm{Na}$ Tabela 4 verificam-se os valores do parâmetro $\mathrm{L}^{\star}$ e das

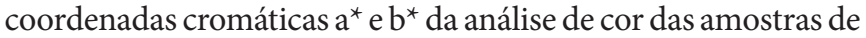
bolo de chocolate. Em relação aos parâmetros de cromaticidade $\left(a^{*}\right.$ e $\left.b^{*}\right)$, foi possível afirmar que todas as amostras se apresentaram nas regiões do vermelha e do amarelo já que a leitura do colorímetro demonstrou valores positivos para estas coordenadas. Silva (2007) menciona que a combinação

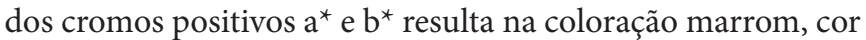
característica de produtos elaborados com chocolate e seus derivados.

Quanto ao parâmetro de luminosidade $\left(\mathrm{L}^{\star}\right)$, todas as amostras foram consideradas escuras, já que na escala de 0 a 100 apresentaram valores abaixo de $50\left(\mathrm{~L}^{\star}<50\right)(\mathrm{COHEN}$; JACKIX, 2005). Os valores de $\mathrm{L}^{\star}$ mais baixos, indicando menor reflectância da luz foram encontrados para as amostras contendo farinha de yacon (A e B), o que pode ser explicado pela presença de compostos fenólicos como o ácido clorogênico e o L-triptofano nas raízes da yacon, tornando-as susceptíveis ao escurecimento enzimático (SEMINARIO; VALDERRAMA; MANRIQUE, 2003).

Outros fatores podem ter afetado a coloração dos bolos. A presença de açúcares e ovos, assim como o calor, acelera reações de caramelização e Maillard, levando ao escurecimento progressivo da crosta e do miolo (GIESE, 2000).

Esteller, Zancanaro Junior e Lannes (2006) formularam bolos de chocolate produzidos com pó de cupuaçu e kerfir e também encontraram valores menores que 50 para a coordenada $\mathrm{L}^{*}$ e valores positivos para as coordenadas de cromaticidade a e b, traduzindo em amostras com coloração mais escura e forte coloração amarelada ou dourada, que, mesmo na coloração castanho-escuro característica de produtos com derivados de chocolate, podem ser "filtradas" e aparecem em produtos ricos em proteínas, açúcares redutores e ovos (carotenoides).

Estudos utilizando o yacon e seus derivados para a formulação de diversos produtos vêm sendo desenvolvidos, no entanto são escassas as informações acerca da caracterização cromática destes.

Tabela 4. Valores obtidos do parâmetro $L^{*}$ e das coordenadas cromáticas $\mathrm{a}^{\star} \mathrm{e}^{\star} \mathrm{da}$ análise de cor das amostras dos bolos.

\begin{tabular}{cccc}
\hline Amostras & $\mathrm{L}^{*}$ & $\mathrm{a}^{*}$ & $\mathrm{~b}^{*}$ \\
\hline $\mathrm{P}$ & $24,39^{\mathrm{a}}$ & $+11,62^{\mathrm{a}}$ & $+14,88^{\mathrm{a}}$ \\
$\mathrm{A}$ & $20,91^{\mathrm{b}}$ & $+9,20^{\mathrm{b}}$ & $+11,67^{\mathrm{b}}$ \\
$\mathrm{B}$ & $19,79^{\mathrm{c}}$ & $+7,25^{\mathrm{c}}$ & $+9,14^{\mathrm{c}}$ \\
\hline
\end{tabular}

Médias seguidas de letras iguais na vertical não diferem significativamente ao nível de 5\% de significância pelo teste de Duncan.

\section{Conclusões}

De acordo com os resultados, foi possível concluir que a adição de 20 e $40 \%$ de farinha de yacon nos bolos de chocolate influenciou no perfil sensorial e na caracterização cromática, deixando-os mais escuros. O bolo com maior teor de farinha de yacon obteve as maiores notas para os atributos: aroma, gosto doce, maciez, sabor de chocolate e qualidade global.

Em adição a sua promissora aceitabilidade, os bolos formulados com farinha de yacon possuem a vantagem de conter maior teor de fibra alimentar na forma de frutanos, que possuem ação prebiótica e aos quais são atribuídos vários benefícios à saúde.

\section{Referências bibliográficas}

ANZALDUA-MORALES, A. La Evalucación Sensorial de los Alimentos en la Teoria y la práctica. Zaragoza: Editorial Acribia, 1994. p. 21-23.

BIEDRZYCKA, E.; BIELECKA, M. Prebiotic effectiveness of fructans of different degrees of polymerization. Trends Food Sciece Technology, v. 15, p. 170-175, 2004.

BRASIL. Ministério da Saúde. Agência Nacional de Vigilância Sanitária. Métodos Físico-químicos para análise de alimentos. Brasília, DF, 2005. p. 311-313.

COHEN, K. O.; JACKIX, M. N. H. Estudo do liquor de cupuaçu. Revista Ciência e Tecnologia de Alimentos, v. 25, n. 1, 2005.

DUTCOSKY, S. D. Análise Sensorial de Alimentos. 2. ed. Curitiba: Champagnat, 2007.

ESTELLER, M. S.; ZANCANARO JUNIOR, O.; LANNES, S. C. S. Bolo de "chocolate" produzido com pó de cupuaçu e kefir. Revista Brasileira de Ciências Farmacêuticas, v. 42, n. 3, 2006.

FRANCK, A. Technological functionality of inulin and oligofructose. British Journal of Nutrition, v. 87, p. 287-291, 2002.

GIESE, J. Color measurement in food as a quality parameter. Food and Technology, v. 54, n. 2, p. 62-63, 2000.

GOTO, K. et al. Isolation and structural analysis of oligosaccharides from yacon (Polymnia sonchifolia). Bioscience Biotechnology and Biochemistry, v. 59, p. 2346-2347, 1995.

HARTEMINK, R.; VANLAERE, K. M. J.; ROMBOUTS, F. M. Growth of enterobacteria on fructo-oligosaccharides. Journal of Applied Microbiology, v. 383, p. 367-374, 1997.

HONG, S. S. U. et al. Melampolides from the Leaves of Smallanthus sonchifolius and Their Inhibitory Activity of LPS-Induced Nitric Oxide Production". Chemical \& Pharmaceutical Bulletin, v. 56, p. 199-202, 2008.

MADRIGAL, L.; SANGRONIS, E. La inulina y derivados como ingredientes claves en alimentos funcionales. Archivos Latinoamericanos de Nutrición, v. 57, n. 4, p. 387-396, 2007.

MANRIQUE, I.; PÁRRAGA, A. Conservación y uso de la biodiversidad de raíces y tubérculos Andinos: Una década de investigación para el desarrollo (1993-2003). Jarabe de yacón: principios y procesamiento. Centro Internacional de La Papa, Lima, 2005. 40 p.

McGUIRE, R. G. Reporting of objective color measurements. Horticultural Science, v. 27, n. 12, p. 1254-1555, 1992.

MINIM, V. P. R. Análise sensorial: estudos com consumidores. Viçosa: Ed. UFV, 2006. 225 p. 
MOSCATTO, J. A. et al. The optimizacion of the formulation for a chocolate cake containg inulin and yacon meal. Intern. Journal of Food and Technology, v. 41, p. 181-188, 2006.

ROBERFROID, M. B. Inulin-Type Fructans: Functional Food Ingredients. The Journal of Nutrition, v. 137, p. 2493-2502, 2007.

ROBERFROID, M. Functional foods: concepts and applications to inulin and oligofructose. British Journal of Nutrition, v. 87, p. 139-143, 2002.

RODRÍGUEZ, R. et al. Dietary fiber from vegetable products as source of functional ingredients. Trends of Food Science and Technology, v. 17, p. 3-15, 2006.

SEMINARIO, J.; VALDERRAMA, M.; MANRIQUE, I. El yacón: fundamentos para el aprovechamiento de un recurso promisorio. Centro Internacional de la Papa - CIP, Lima, 2003.

SILVA, A. S. S. et al. Frutoligossacarídeos: fibras alimentares ativas. Boletim do CEPPA, v. 25, n. 2, p. 295-304, 2007.
SILVA, A. S. S. Propriedades tecnológicas e sensoriais de pães confeccionados com diferentes quantidades de yacon. In: SILVA, A. S. S. A raíz da yacon (Smallanthus sonchifollius Poepping \& Endlicher) como fonte de fibras alimentares, sua caracterzação físico-química, uso na panificação e sua influência na glicemia pós-prandial. 2007. Tese (Doutorado em Ciências dos Alimentos)Universidade Federal de Santa Catarina, Santa Catarina, 2007. cap. 3, p. 101-131.

STATSOFT. STATISTICA for Windows 6.0. Tulsa-UK: StatSoft, 2002. Computer program manual.

ZULETA, A.; SAMBUCETTI, M. E. Fructanos: características estructurales y metodologia analítica. In: LAJOLO, F. M.; MENEZES, E. W. (eds). Carbohidratos en Alimentos Regionales Iberoamericanos. São Paulo: Editora da Universidade de São Paulo, 2006. 648 p. 